\title{
Patient education: Preventing diabetic foot infections
}

\author{
Pavelina Mihaela Andrei', Maria Daniela Tanasescu ${ }^{1,2}$, Pavel Minca ${ }^{1,2}$, \\ Andra-Elena Balcangiu-Stroescu, ${ }^{1,2}$, Alexandra Maria Limbau', \\ Constantin lonescu Tirgoviste ${ }^{1,3}$ \\ 1"Carol Davila" University of Medicine and Pharmacy, Faculty of Medicine, Bucharest, Romania \\ 2Department of Internal Medicine, University Emergency Hospital, Bucharest, Romania \\ 3"N.C. Paulescu" National Institute of Diabetes, Nutrition and Metabolic Diseases, Bucharest, Romania
}

\begin{abstract}
Diabetic foot infections are a common complication in people with diabetes. This article will discuss the foot-related complications that can happen in people with diabetes, as well as guidelines for good foot care. Patient education is a central component in the self management of diabetes infections in both prevention and treatment. Poor knowledge of foot care practices is an important risk factor for foot problems among diabetics. Studies have demonstrated deficiencies in knowledge and practices regarding foot care, particularly among rural populations with diabetes. Patients should be informed about the risk factors and their complications that could lead to amputation. The mainstay therapy for intervention of diabetic lower limb complications is prevention.
\end{abstract}

Keywords: diabetic foot, infections, foot care, prevention

\section{INTRODUCTION}

Diabetic foot infections are associated with substantial morbidity and mortality. Over time, diabetes can lead to various complications, many of which can be serious if they are not identified and addressed promptly (1). Important risk factors in the development of diabetic foot infections include neuropathy, peripheral vascular disease, and poor glycemic control. Educating patients about proper foot care and periodic foot examinations are effective interventions to prevent ulceration. Regular foot exams to check for problems or changes are a critical part of managing diabetes (2). Trauma in patients with one or more of these risk factors precipitates development of wounds that can be slow to heal and predispose to secondary infection.

It may help to make the self foot exams a part of daily routine. This should include looking carefully at all parts of the feet, especially the area between the toes (3). Look for excessive skin dryness, scaling, broken skin, ulcers, blisters, areas of increased warmth or redness, or changes in callus formation. Nerve damage may lead to unusual sensations in the feet and legs, including pain, burning, tingling, or numbness (4). A weak pulse, cold feet, thin skin, and lack of hair in the area suggest that there is reduced blood flow.

\section{DIABETIC FOOT INFECTIONS}

All patients with diabetes should be examined to identify risk for foot ulceration. The feet should be visually inspected at each routine visit to identify problems with nail care, poorly fitting footwear resulting in barotrauma, fungal infections, and callus formation that may result in more severe foot problems. A comprehensive foot examination should be performed annually on patients with diabetes to identify risk factors predictive of ulcers and amputation; and should include inspection, assessment of pedal pulses, and testing for loss of protective sensation. 
Diabetic foot infections can develop as a result of neuropathic or ischemic ulcers, traumatic wounds, skin cracks or fissures, or other defects in the skin of the foot or nail beds (paronychia). Thus, infection can present as localized superficial skin involvement at the site of a preexisting lesion or as an infection of the skin or deeper skin structures that has spread beyond the site of local trauma. Such infections can subsequently extend to joints, bones, and the systemic circulation.

The microbiology of diabetic foot wounds is variable depending on the extent of involvement. Superficial diabetic foot infections (including cellulitis and infected ulcers) are likely due to aerobic gram-positive cocci including Staphylococcus aureus, Streptococcus pyogenes (5). It is also important to note that diabetic patients with chronic foot wounds who receive repeated and prolonged courses of antibiotics represent an important risk group for development of vancomycin-intermediate $\mathrm{S}$. aureus infections. Ulcers that are deep, chronically infected, are more likely to be polymicrobial. Such wounds may involve enterococci, Enterobacteriaceae, Pseudomonas aeruginosa, and anaerobes. Wounds with extensive local inflammation, cutaneous bullae, severe ischemia, necrosis, malodorous drainage, or gangrene with signs of systemic toxicity should be presumed to have anaerobic organisms in addition to the above pathogens and may denote the presence of a limb-threatening infection. Potential pathogens include anaerobic streptococci, Bacteroides species, and Clostridium species. Systemic signs such as fever, chills, hypotension, and tachycardia may accompany local signs of infection, and their presence indicates an increased severity of infection. The possibility of osteomyelitis should be considered in diabetic patients with foot wounds associated with signs of infection in the deeper soft tissues and in patients with chronic ulcers, particularly those overlying bony prominences that do not heal after several weeks of wound care (6). Clinical features associated with underlying osteomyelitis in patients with diabetic foot ulcers include ulcer size $>2 \mathrm{~cm}^{2}$ and depth allowing visibly exposed bone or ability to probe to bone (7).

\section{DIAGNOSIS OF DIABETIC FOOT INFECTIONS}

The diagnosis of a diabetic foot infection is primarily based on suggestive clinical manifestations. Systemic signs such as fever, chills, hypotension, and tachycardia may accompany local signs of infection, and their presence indicates an increased severity of infection. The presence of two or more features of inflammation (erythema, warmth, tenderness, swelling, induration and purulent secretions) can establish the diagnosis. As many diabetic foot wounds are colonized by bacteria, the presence of microbial growth from a wound culture in the absence of supportive clinical findings is not sufficient to make the diagnosis of infection. Clinical examination should also include a neurologic evaluation that documents the extent of sensory loss as well as a vascular evaluation of the presence and severity of arterial and/or venous insufficiency (8). Infection may coexist with other inflammatory processes, and empiric antimicrobial therapy may be warranted in some cases when the diagnosis is unclear. These include trauma, crystal-associated arthritis, acute Charcot arthropathy, fracture, thrombosis, and venous stasis.

Laboratory evaluation should include complete blood count as well as measurement of blood glucose, electrolytes, renal function and subsequent inflammatory markers such as erythrocyte sedimentation rate (ESR) and C-reactive protein (CRP). Some studies, but not all, have suggested that procalcitonin (PCT), a novel inflammatory marker, may also be useful if laboratory facilities that test this substance are locally available; further investigation is needed to determine the clinical utility of this assay (9).

Physical examination findings such as diminished pulses, decrease in skin temperature, thin skin, lack of skin hair, and bluish skin color are not specific enough to guide further management in an individual patient (10). Patients with clinical evidence of peripheral artery disease should have ankle-brachial index testing. A low ankle-brachial index in the presence of a foot ulcer suggests that the prognosis will be improved with reconstructive vascular surgery (11).

Sweating is diminished or absent; as a result, the skin of the feet remains dry and has a tendency to become scaly and cracked, thereby allowing infection to penetrate below the skin. Lack of autonomic tone in the capillary circulation causes shunting of blood from arteries directly into veins, bypassing the tissues that need nutrition (12). This results in a foot that feels warm and has distended veins and bounding pulses (13). Despite these apparent signs of adequate perfusion, the foot is vulnerable to local ,microvascular” gangrene, will heal very poorly and slowly, and will be less able to resist infection (4).

Neuropathy, promotes ulcer formation by decreasing pain sensation and perception of pressure, by causing anatomic deformities, and by impairing 
the microcirculation and the integrity of the skin. Once ulcers form, healing may be delayed or difficult to achieve, particularly if infection penetrates to deep tissues and bone and/or there is diminished local blood flow. Bony deformities, joint mobility, and gait and balance should also be assessed. This leads to subluxation of the proximal interphalangeal-metatarsal joints, resulting in a claw toe appearance. One consequence of this abnormality is increased pressure on the metatarsal heads, which are a common site of ulcer development.

A later complication is Charcot arthropathy, which is characterized by collapse of the arch of the midfoot and abnormal bony prominences (14). These changes are often triggered by injury, inflammation, and hyperperfusion, accompanied by the triad of small muscle wasting, decreased sensation, and abnormal distribution of weight when standing, leading to repetitive microtrauma due to the absence of protective pain sensation.

\section{GENERAL MEASURES}

There are several things that patients can do to reduce foot complications. Careful management of blood sugar levels can reduce the risk of circulation problems and nerve damage that often lead to diabetic foot complications. Smoking can worsen heart and vascular problems and reduce circulation to the feet. They need to avoid activities that can injure the feet and use care when trimming their nails. Weight control/dietary modification, reduction in alcohol, exercise are other things that modification lifestyle changes (10).

Several risk factors are predictive of ulcers and amputation. Early recognition and management of risk factors is important for reducing morbidity of foot ulceration. Most are readily identifiable from the history or physical examination. The most important risk factors are: previous foot ulceration, neuropathy (loss of protective sensation), foot deformity, vascular disease.

The skin should be assessed for integrity, especially between the toes and under the metatarsal heads. Findings that may herald a developing foot ulcer include the following: lesions between adjacent toes due to pressure from tight shoes cramming them together, macerated areas between the toes (,,athlete's foot”); these lesions are often painless and may go unnoticed until bacterial infection supervenes, callused areas. The presence of erythema, warmth, or fissures may indicate areas of tissue damage.

\section{BASIC PRINCIPLES OF WOUND MANAGEMENT}

Management of diabetic foot infections requires attentive wound management, good nutrition, appropriate antimicrobial therapy, glycemic control, and fluid and electrolyte balance. Local wound care for diabetic foot infections typically includes debridement of callus and necrotic tissue, wound cleansing, and relief of pressure on the ulcer. Topical agents such as antiseptics and antimicrobial agents can be used to control locally heavy contamination. Appling a suitable dressing to a wound, can arguably have a significant impact on the speed of wound healing, wound strength and function of the repaired skin, and cosmetic appearance of the resulting scar. The purpose of wound dressing is to absorb exudate and create a moist environment to promote healing. A wide array of dressing and wound healing products for ulcer management has been developed. These products include enzymes, gels, hydrocolloids and antiseptics containing iodine or silver salts.

Empiric antibiotic therapy should be selected based on the severity of infection and the likelihood of involvement of resistant organisms. Patients with mild infection should receive oral antibiotic therapy in conjunction with attentive wound care. The antibiotics chosen and duration of therapy depend upon the depth and severity of infection. For severe infections, patients require surgical debridement after receiving intravenous antibiotic therapy perioperatively (15). Surgical debridement is required for cure of infections complicated by abscess, extensive bone or joint involvement, crepitus, necrosis, gangrene or necrotizing fasciitis and is important for source control in patients with severe sepsis. In addition to surgical debridement, revascularization (via angioplasty or bypass grafting) and/or amputation may be necessary (3).

\section{RECOMMENDATIONS FOR PATIENTS}

In conjunction with the comprehensive foot exam, advice for prophylactic foot care should be given to all patients (16). These recommendations are particularly important in patients with existing neuropathy: avoid smoking, avoid going barefoot, even at home, and especially on hot decks and hot sand, trim toenails to shape of the toe, and remove sharp edges with a nail file; do not cut cuticles (17), wash in lukewarm water, dry thoroughly (including between the toes), and check feet daily, test water temperature before stepping into a bath, socks 
should fit and be changed daily, shoes should be snug, but not tight, and customized if feet are misshapen or have ulcers.

Patient education must also emphasize the need to reduce the risk of avoidable damage to the skin. Integrity of the skin is essential because it forms a protective barrier (18). Once breached, it allows ingress by pathogenic bacteria, with consequent risk of spreading infection, inflammation, thrombotic occlusion of small and medium-sized vessels and gangrene (19). Lifestyle modification should be discussed: weight control/dietary modification, reduction in alcohol intake, cessation of smoking, proper exercises.

Patient education is a central component in the self management of diabetes complications in both prevention and treatment. It is one of the major management strategies for prevention and treatment of diabetic foot ulcer (20). Patient involvement is vital for the successful care of diabetic foot ulcers. The primary task of the health care team is to boost patient's self confidence, improve patient awareness and provide support. Poor knowledge of foot care practices is an important risk factor for foot problems among diabetics. Patients should be informed about the risk factors and their complications that lead to amputation. The mainstay therapy for intervention of diabetic lower limb complications is prevention.

The advice of a psychologist may help to deal with problems of denial of the condition, particularly in the later stages of neuropathy (21).

\section{CONCLUSIONS}

Patients should be informed about the risk factors and their complications that could lead to amputation. The mainstay therapy for intervention of diabetic lower limb complications is prevention.

\section{Acknowledgement}

All authors equally contributed to the present paper.

\section{REFERENCES}

1. Barth R, Campbell LV, Allen S, Jupp JJ, Chisholm DJ. Intensive education improves knowledge, compliance, and foot problems in type 2 diabetes. Diabet Med. 1991 Feb-Mar; 8(2):111-7.

2. van Netten JJ, Lazzarini PA, Armstrong DG, Bus SA, Fitridge R, Harding $\mathrm{K}$ et al. Diabetic Foot Australia guideline on footwear for people with diabetes. J Foot Ankle Res. 2018; 11(1):2.

3. Hurlow JJ, Humphreys GJ, Bowling FL, McBain AJ. Diabetic foot infection: A critical complication. Int Wound J. 2018; 15(5):814-21.

4. Singh N, Armstrong DG, Lipsky BA. Preventing foot ulcers. J Am Med Assoc. 2005; 293(2):94-6.

5. Lipsky BA, Berendt AR, Deery HG, Embil JM, Joseph WS, Karchmer AW et al. Diagnosis and treatment of diabetic foot infections. J Am Podiatr Med Assoc. 2005; 95(2):885-910.

6. Butalia S, Palda VA. Does This Patient With Diabetes Have Osteomyelitis of the Lower Extremity? JAMA. 2013;299(7).

7. Johani K, Fritz BG, Bjarnsholt T, Lipsky BA, Jensen SO, Yang M et al. Understanding the microbiome of diabetic foot osteomyelitis: insights from molecular and microscopic approaches. Clin Microbiol Infect. 2019; 25(3):332-9.

8. Tesfaye S, Chaturvedi N, Eaton SEM, Ward JD, Manes C, lonescu-Tirgoviste $\mathrm{C}$ et al. Vascular risk factors and diabetic neuropathy. N Engl J Med. 27 ianuarie 2005; 352(4).

9. Velissaris D, Pantzaris ND, Platanaki C, Antonopoulou N, Gogos C. Procalcitonin as a diagnostic and prognostic marker in diabetic foot infection. Rom J Intern Med. 2018 Mar 1;56(1):3-8.

10. Brod M. Quality of life issues in patients with diabetes and lower extremity ulcers: patients and care givers. Qual Life Res. 1998; 7(4):365-72.

11. Bălăceanu $A$, Diaconu $C$, Sarsan $A$. Indicele gleznă-braţ în evaluarea pacienţilor cu arteriopatie obliterantă cronică a membrelor inferioare. Med Mod. 2010; XVII(12):632-4.
12. Malgrange $D$. Physiopathology of the diabetic foot - Physiopathologie du pied diabetique. Rev Med Interne. 2008; 29(SUPPL. 2):S231-7.

13. Pecoraro RE, Reiber GE, Burgess EM. Pathways to diabetic limb amputation: Basis for prevention. Diabetes Care. 1990; 13(5):513-21.

14. Wanzou JPV, Sekimpi P, Komagum JO, Nakwagala F, Mwaka ES. Charcot arthropathy of the diabetic foot in a sub-Saharan tertiary hospital: A cross-sectional study. J Foot Ankle Res. 2019; 12(1):1-9.

15. Burdette-Taylor M. Prevent Wounds by Conducting a Comprehensive Foot Examination and Intervention. Healthcare. 2015; 3(3):586-92.

16. Bodman MA, Dulebohn SC. Diabetic Neuropathy. StatPearls. 2017.

17. Takehara K, Oe M, Tsunemi Y, Nagase T, Ohashi Y, lizaka S et al. Factors associated with presence and severity of toenail onychomycosis in patients with diabetes: A cross-sectional study. Int J Nurs Stud. 2011; 48(9):1101-8.

18. Demirseren DD, Emre S, Akoglu G, Arpacı D, Arman A, Metin A et al. Relationship between skin diseases and extracutaneous complications of diabetes mellitus: Clinical analysis of 750 patients. Am J Clin Dermatol. 2014; 15(1):65-70.

19. Altay FA, Kuzi S, Altay M, Ateş I, Gürbüz Y, Tütüncü EE et al. Predicting diabetic foot ulcer infection using the neutrophil-tolymphocyte ratio: a prospective study. J Wound Care. 2019; 28(9):601-7.

20. Lim JZM, Ng NSL, Thomas C. Prevention and treatment of diabetic foot ulcers. J R Soc Med. 2017; 110(3):104-9.

21. Gunes AE, Cimsit M. Can amputation be prevented in diabetic foot? Interdisciplinary approach to diabetic foot: A case report. Undersea Hyperb Med. 2017; 44(2):157-60. 